\title{
Estrutura Interna da Versão Brasileira do Questionário de Estresse Traumático Secundário
}

\author{
Patricia Dalagasperina ${ }^{1}$ \\ Elisa Kern de Castro ${ }^{2}$ \\ Roberto de Moraes Cruz' \\ Artur Pereira ${ }^{3}$ \\ Bernardo Moreno Jiménez ${ }^{4}$ \\ ${ }^{1}$ Universidade Federal de Santa Catarina, Florianópolis, Santa Catarina, Brasil \\ ${ }^{2}$ Universidade Lusíada de Lisboa, Lisboa, Portugal \\ ${ }^{3}$ Fundação Getúlio Vargas, Rio de Janeiro, Rio de Janeiro, Brasil \\ ${ }^{4}$ Universidade de Salamanca, Salamanca, Espanha
}

\begin{abstract}
Resumo
O Estresse Traumático Secundário caracteriza-se por um conjunto de sintomas e condutas que se manifestam após a exposição indireta ao trauma. Profissionais que cuidam de pessoas traumatizadas estão susceptíveis ao transtorno. Este estudo examinou a estrutura interna da versão brasileira do Cuestionario de Estrés Traumático Secundario. Participaram 624 profissionais da saúde, acessados on-line. Para realização das análises fatoriais, a amostra foi dividida em dois grupos. Na análise exploratória, foram retidos 12 componentes, com cargas fatoriais entre 0,312 e 0,999 . Os resultados da análise confirmatória revelaram que o modelo de doze fatores para 50 itens forneceu o melhor ajuste possível para os dados (RMSEA = 0,044; IC = 10\%-90\%; CFI = 0,949; TLI $=0,904)$ e índice de consistência interna geral do modelo foi de 0,91 . Esses doze fatores foram distribuídos em quatro escalas (Antecedentes, Síndrome de Trauma Secundário, Personalidade e Consequências), com índices psicométricos relativamente baixos e uma nova organização dos itens das escalas do QETS, em contraste com a versão teórica original do instrumento. Sugere-se que novos estudos psicométricos analisem as escalas do questionário, separadamente, e investiguem outras categorias profissionais, especialmente na área dos serviços de emergências, além de estudos comparativos com amostras clínicas.

Palavras-chave: estresse, trauma emocional, testes psicológicos, validade
\end{abstract}

\section{Internal Structure of the Secondary Traumatic Stress Questionnaire in Brazil}

\section{Abstract}

Secondary Traumatic Stress is characterized by a set of symptoms and behaviors that manifest themselves after the indirect exposure to the trauma. Professionals caring for traumatized people are susceptible to the disorder. This study examined the internal structure of the Brazilian version of the Cuestionario de Estrés Traumático Secundario. 624 health professionals participated, accessing the online questionnaire. To perform the factorial analysis, the sample was divided into two groups. In the exploratory analysis, 12 components were retained, with factorial loads between 0.312 and 0.999 . The confirmatory analysis revealed a twelve-factor model for 50 items provided the best possible fit for the data $(\mathrm{RMSEA}=0.044 ; \mathrm{CI}=10 \%-90 \%$; CFI $=0.949$; TLI $=0.904)$. However, at the end of the data discussion, 48 items remained in the model, and the overall internal consistency index of the model was 0.91. These twelve factors were distributed on four scales (Background, Secondary Trauma Syndrome, Personality and Consequences), with relatively low psychometric indices and a new organization of the items on the QETS scales in contrast to the theoretical framework in the the original instrument. It is suggested that new psychometric studies analyze the scales of the questionnaire separately and investigate other professional categories, especially in the area of emergency services and comparative studies with clinical samples.

Keywords: stress, emotional trauma, psychological tests, validity

\section{Estructura Interna de la Versión Brasileña del Cuestionario de Estrés Traumático Secundario}

\section{Resumen}

El estrés traumático secundario se caracteriza por un conjunto de síntomas y conductas que se manifiestan después de la exposición indirecta al trauma. Los profesionales que cuidan a las personas traumatizadas son susceptibles al trastorno. Este estudio examinó estructura interna de la versión brasileña del Cuestionario de Estrés Traumático Secundario. Participaron 624 profesionales de la salud, accediendo al cuestionario online. Para la realización de los análisis factoriales la muestra fue dividida en dos grupos. En el análisis exploratorio, se retuvieron 12 componentes, con cargas factoriales entre 0.312 y 0.999 . El análisis confirmatorio reveló que el modelo de doce factores para 50 ítems proporcionó el mejor ajuste posible para los datos (RMSEA $=0.044 ; \mathrm{IC}=10 \%-90 \% ; \mathrm{CFI}=0.949 ; \mathrm{TLI}=0.904)$. Sin embargo, al final de la discusión de los datos quedaron 48 ítems en el modelo. El índice de consistencia interna general del modelo fue de 0,91 . Estos doce factores se distribuyeron en cuatro escalas (Antecedentes, Síndrome de Trauma Secundario, Personalidad y Consecuencias), con índices psicométricos relativamente bajos y una nueva organización de los ítems en las escalas QETS, en contraste con la versión teórica original del instrumento. Se sugiere que nuevos estudios psicométricos analizan las escalas del cuestionario por separado e investiguen otras categorías profesionales, especialmente en el área de los servicios de emergencias y estudos comparativos con amostras clínicas. Palabras clave: estrés, trauma emocional, tests psicológicos, validez 


\section{Introdução}

O termo Estresse Traumático Secundário (ETS) foi proposto, em 1983, por Charles Figley, para designar o sofrimento que está presente no ato de cuidar de pessoas traumatizadas. Existiria um "custo no cuidar", representado por um conjunto de emoções e de condutas que se manifestam após a exposição ao trauma vivenciado por outra pessoa (Figley, 1983). O termo "secundário" se refere àquelas pessoas que testemunham um evento traumático, que prestam serviços ou que convivem com pessoas que foram vítimas de trauma, ou seja, familiares, cônjuges, amigos e profissionais que fornecem algum tipo de cuidado às vítimas estão em risco de desenvolver ETS (Figley, 1995).

Ao investigar o ETS em profissionais da saúde entende-se, neste estudo, que a vítima secundária (profissional da saúde) sofre de ETS, enquanto a vítima primária (paciente) desenvolve o Transtorno de Estresse Pós-Traumático (TEPT) (Moreno-Jiménez, Morante, Rodríguez, \& Garrosa, 2004a; Pedras \& Pereira, 2012). A atual definição do TEPT considera que esse transtorno pode ocorrer em ambas as formas de exposição ao trauma (primária e secundária), conforme descrito no Critério A1 do TEPT, no DSM-V (APA, 2013). Há que considerar, contudo, que a vivência direta ou indireta do trauma pode ter singularidades. O ETS está relacionado ao contexto de trabalho, onde os profissionais prestam cuidados e ajuda às vítimas de traumas (Figley, 1995; Moreno-Jiménez et al., 2004a). Esses profissionais têm clareza que o seu ofício envolve a exposição constante a diferentes eventos traumáticos (Hensel, Ruiz, Finney, \& Dewa, 2015), o que indica a possibilidade de intervir de forma preventiva.

No TEPT, o evento traumático ocorre de forma súbita e inesperada reduzindo as possibilidades de ações preventivas. Além disso, o transtorno não se relaciona apenas a populações específicas, como no caso dos profissionais da saúde, podendo acometer as pessoas em todas as faixas etárias (APA, 2013). Para o desenvolvimento do TEPT não é necessário possuir habilidades empáticas. Já, no ETS, essa habilidade é central para o seu surgimento (Figley, 1995; Newell \& MacNeil, 2010).

Ao longo das investigações sobre o trauma, desde a década de 1980, diferentes terminologias foram utilizadas para se referir ao ETS. Termos como "Fadiga de Compaixão" (FC) e "Trauma Vicário" (TV) estão entre eles (Figley, 1995; Moreno-Jiménez, Cobo, \& Blanco, 2013; Pedras \& Pereira, 2012). Além destes, a Síndrome de Burnout (SB) possui algumas semelhanças com o ETS, conforme Pedras \& Pereira (2012). Alguns dos sintomas similares encontrados em cada um desses termos têm gerado uma certa confusão na literatura quanto às especificidades de cada construto (Moreno-Jiménez et al., 2013), ainda que as tentativas de esclarecimento no uso de cada uma das terminologias permaneçam atual (Cieslak, 2014; Newell \& MacNeil, 2010).

No Brasil, estudos que visam identificar o sofrimento dos trabalhadores da saúde ainda são escassos. A produção científica nacional sobre a SB aponta que os professores e os profissionais da saúde representam as categorias profissionais mais pesquisadas (Carlotto \& Câmara, 2008). O termo "Fadiga de Compaixão" foi citado em apenas quatro publicações no Brasil (Barbosa, Souza, \& Moreira, 2014; Galiana, Arena, Oliver, Sansó, \& Benito, 2017). A relação do TEPT com os profissionais da saúde foi mencionada em um único artigo (Lima \& Ávila, 2011). E, por fim, os termos "Trauma Vicário" e "Estresse Traumático Secundário", até o momento, não foram apontados em nenhum estudo científico brasileiro.

A gênese do ETS entre os profissionais da ajuda tem sido explicada por diferentes modelos teóricos. Em 1995, Figley propôs um modelo relacionado aos profissionais, composto por quatro fatores: a empatia, o comportamento manifestado pela vítima, as dificuldades para distanciar-se do trabalho e o sentimento de satisfação por oferecer ajuda. O modelo de ETS proposto por Dutton e Rubistein (1995) engloba: o evento traumático, as reações frente a isso, as estratégias de enfrentamento e o contexto ambiental e pessoal. Beaton e Murphy (1995) descreveram um modelo semelhante ao anterior, porém destacaram o papel dos fatores organizacionais e de determinadas características de personalidade, como elementos mediadores, que podem potencializar o ETS.

Entre as medidas psicométricas comumente encontradas na literatura para avaliação do estresse traumático em vítimas expostas indiretamente ao trauma estão: Professional Quality of Life Scale (ProQOL) e Secondary Traumatic Stress Scale (STSS). A ProQOL é a mais utilizada para determinar qualidade de vida de profissionais dos serviços de cuidados, possuindo mais de 200 citações (Perkins \& Sprang, 2013). Os 30 itens que a compõem estão divididos em três subescalas que apresentaram alta confiabilidade: SC $(0,88) ; \mathrm{SB}(0,75)$ e Trauma/FC $(0,81)$. No âmbito nacional, estudos de validação desse instrumento apontaram estimativas de confiabilidade adequadas. A análise de consistência dos 
fatores, investigado em profissionais da saúde, apresentou validade confiável, sendo os valores de alfas: 0,81 para SB; 0,83 , para Trauma/FC e 0,76 para SC (Lago $\&$ Codo, 2013). No estudo transcultural que avaliou as propriedades psicométricas do instrumento, nas versões em português (Brasil) e em espanhol (Espanha), as dimensões SC e o Trauma/FC apresentaram índices significativos de confiabilidade. Na amostra de brasileiros, os alfas foram 0,85 para SC e 0,77 para o Trauma/ FC. Entre os espanhóis, os resultados foram, respectivamente, 0,77 e 0,78 . No entanto, as estimativas para a dimensão SB indicaram problemas de confiabilidade em ambas as amostras, com um alfa de 0,65 na versão portuguesa e de 0,53 na versão espanhola (Galiana et al., 2017).

A Secondary Traumatic Stress Scale (STSS) foi desenvolvida para mensurar especificamente os sintomas de trauma secundário em profissionais que fornecem cuidados. A STSS avalia a frequência de sintomas experimentados na última semana, usando um formato de resposta de tipo Likert de 5 pontos, congruente com a definição DSM-IV do TEPT. Seus 17 itens dividem-se nas subescalas Intrusão, Evitação e Excitabilidade. A STSS apresenta propriedades psicométricas satisfatórias que incluem alta confiabilidade interna de 0,93 a 0,95 para a escala geral (Kintzle, Yarvis, \& Bride, 2013), validade convergente e discriminante e validade fatorial e diferentes formas de pontuação (Bride, Radey, \& Figley, 2007). Comparativamente, o ProQOL mensura a qualidade de vida profissional com base em três fatores, compreendendo o trauma secundário como uma faceta da FC, enquanto a STSS avalia somente os sintomas do ETS.

Praticamente uma década após afirmação de modelos sobre o Estresse Traumático Secundário em profissionais de cuidado, Moreno-Jiménez, Morante, Rodriguez e Garrosa (2004b) promoveram uma nova abordagem teórica, denominada de modelo processual do ETS. Nesse modelo, são consideradas: as variáveis sociodemográficas; os antecedentes organizacionais referentes às dificuldades inerentes à tarefa e ao nível de satisfação em desempenhá-las; as variáveis de personalidade, com ênfase na empatia, na compreensão, no desafio e no senso de humor; e as consequências individuais, sociais ou organizacionais. Nesse entendimento, considera-se um conjunto de três fatores para caracterizar os sintomas do ETS: a Fadiga Emocional, a Mudança de Crenças e a Sintomatologia Traumática Secundária.
O instrumento que mensura as variáveis do modelo processual do ETS, que será analisado neste estudo, denomina-se Cuestionario de Estrés Traumático Secundario (CETS), formado por quatro escalas: Antecedentes, Trauma Secundário, Personalidade e Consequências. A CETS demonstrou propriedades psicométricas satisfatórias, em uma amostra de 204 profissionais de emergência e 223 bombeiros no contexto mexicano (Lara, Jiménez, Muñoz, Merino, \& Chávez, 2011). Nesse estudo, por meio de análise fatorial exploratória (AFE), foi encontrada uma estrutura de três fatores que corresponderam ao seu fundamento teórico (fadiga emocional, sintomas de trauma secundário e abalo de crenças). Além disso, foi realizada uma análise fatorial confirmatória (AFC) que identificou um modelo de três fatores correlatos à AFE e, nesse caso, a confiabilidade das três dimensões do CETS foi examinada por meio do alfa de Cronbach $(>0,77)$.

Nesse cenário, o CETS apresenta uma proposta diferente ao fornecer a mensuração do processo do ETS, capaz de ampliar a compreensão do fenômeno a partir da mensuração dos sintomas e os fatores a ele associados. Além dessa vantagem, não há outros estudos nacionais que mencionem o uso desse instrumento. Com intuito de ampliar a compreensão do ETS entre os profissionais da saúde, este estudo se propôs a explorar a estrutura interna do QETS, por meio de um estudo exploratório e confirmatório das propriedades psicométricas do instrumento e do modelo teórico que o sustenta.

\section{Método}

\section{Participantes}

Participaram da pesquisa 624 profissionais da saúde (enfermeiros, técnicos de enfermagem, médicos, psicólogos, fisioterapeutas e outros) de diferentes instituições do país. O cálculo amostral considerou um número ideal de dez participantes para cada item da escala analisada (63 itens) (Hair, Anderson, Tatham, \& Black 2007). Para a investigação da estrutura interna, foram realizadas análises fatoriais exploratória e confirmatória, com a divisão da amostra em dois grupos, com 312 participantes cada, respectivamente (Grupo A e B).

O grupo A, utilizado para a análise fatorial exploratória (AFE), caracterizou-se pela predominância do público feminino $(88,1 \%)$, sendo mais da metade dos participantes casados ou em união estável (55,1\%). A maioria é composta de técnicos de enfermagem (40,1\%), que atuam na região sul do país $(59,9 \%)$ e tem vínculo 
empregatício fixo $(87,18 \%)$. A idade dos participantes variou entre 18 e 68 anos, com média de idade de 34,1 $(D P=9,1)$ e o número de horas trabalhadas alternou entre 8 e 72 horas, com média de 40 horas semanais.

Os dados sociodemográficos e laborais do grupo $\mathrm{B}$, no qual empregou-se a análise fatorial confirmatória (AFC), são similares aos da AFE. A maioria dos profissionais é do sexo feminino (91,35\%), casada ou em união estável $(56,7 \%)$ e técnicos de enfermagem $(41,0 \%)$. Mais da metade atuam na região sul do país $(53,8 \%)$ e possuem vinculo fixo de trabalho. A média de horas trabalhadas semanalmente foi de 39,7 e a média de idade da amostra foi de $34,4(D P=9,13)$.

\section{Instrumentos}

Foram utilizados os seguintes instrumentos

a) Questionário de Dados Sociodemográficos e Laborais. Investigou características, como: faixa etária, grau de escolaridade, gênero, estado civil, número de horas de trabalho semanais, tempo experiência na profissão.

b) Post Traumatic Stress Disorder - Checklist - Civilian Version (PCL-C) adaptado para o português do Brasil (Berger, Mendlowicz, Souza, \& Figueira, 2004). É um instrumento de autorrelato formado por 17 itens, paralelos aos critérios do TEPT estabelecidos pelo DSM-IV, cujas respostas devem ser realizadas em uma escala Likert de 1 (nada) a 5 (muito). O indivíduo apresenta indicativo de TEPT se pontuar ao menos um item do critério B (Revivência), três do critério $C$ (Evitação) e dois itens do critério D (Excitabilidade). Considera-se presente o sintoma quando a resposta pontuar 3 ou mais (ISTSS, 2014).

c) Cuestionário de Estrés Traumático Secundario. Originalmente formado por 63 itens, foi elaborado na Espanha por Moreno et al. (2004a). O questionário está em formato de respostas tipo Likert com quatro alternativas entre: (1) discordo totalmente a (4) concordo totalmente. A avaliação do ETS é mensurada por meio de quatro escalas (Antecedentes, Síndrome de Trauma Secundário, Personalidade e Consequências) e 14 subescalas (Carga horária e laboral, Pressão social, Tarefa traumática, Satisfação por ajudar, Fadiga emocional, Mudança de crenças, Sintomatologia traumática secundária, Compreensibilidade, Desafio, Empatia, Sentido do humor, Consequências físicas, Consequências sociais e Consequências organizacionais).

A adaptação do CETS para o contexto brasileiro foi realizada por meio da busca da validade baseada no conteúdo, com tradução e retrotradução do espanhol para o português e determinação do Coeficiente de Validade de Conteúdo (CVC > 0,92). Posteriormente, foram realizados estudos correlacionais, no contexto brasileiro, com profissionais de Psicologia (Castro, Massome, \& Dalagasperina, 2018), entre as escalas do questionário (antecedentes, síndrome, personalidade e consequências), as dimensões do PCL-C e as variáveis sociodemográficas e laborais. Verificaram-se correlações positivas e moderadas entre a escala de personalidade e a escala de antecedentes $(r=0,520 ; p$ $<0,001)$ e a escala de sintomas traumáticos $(r=0,673$; $p<0,001)$, mas correlação negativa e significativa, de intensidade fraca, entre a personalidade e a idade dos psicólogos $(r=-0,192 ; p<0,05)$. Na versão adaptada para o português do Brasil, denominou-se de Questionário de Estresse Traumático Secundário (QETS).

\section{Procedimentos de Coleta de Dados}

O acesso aos participantes foi por conveniência. O convite aos profissionais da saúde foi divulgado nas redes sociais (Facebook e Linkedin) e nas listas do correio eletrônico dos pesquisadores envolvidos. No convite constavam informações sobre o tema da pesquisa, o público-alvo, o tempo gasto para respondê-la (30 minutos) e um link para acessar a página da pesquisa onde constavam os instrumentos e o Termo de Consentimento Livre e Esclarecido (TCLE).

\section{Procedimentos de Análise de Dados}

Para validação da estrutura original do QETS empregou-se a AFE baseada no método de Análises Paralelas. O estudo da adequabilidade do modelo mensurado ocorreu através da matriz de correlação Kaiser-Meyer-Olkin (KMO) e o teste de esfericidade de Bartlett. Foi realizada a AFE para identificar mínimo número de fatores necessários para explicar o máximo da variância representada pelas variáveis originais. Utilizou-se a rotação Oblimin, considerando que há, na literatura, evidências de que os fatores do QETS estão relacionados entre si (Tabachnick \& Fidell, 2007). Os itens com carga fatorial inferior a 0,30 foram excluídos. Empregou-se o método da análise paralela (Hayton, Allen, \& Scarpello, 2004) para determinar o número de fatores na análise dos componentes principais. As 
análises estatísticas descritivas e inferenciais bivariadas e multivariadas foram realizadas no programa Statistical Package for the Social Sciences (SPSS), versão 20.0.

Empregou-se a AFC, com ajuste por meio de estimadores de máxima verossimilhança (MLE), cujos cálculos foram realizados no software $\mathrm{R}$ 3.3.1. Foi testado o ajuste do modelo para 12 fatores e 50 itens. As estatísticas utilizadas para ajuste ao modelo foram: Root Mean Square Error of Approximation (RMSEA,) Índice de Ajuste Comparativo (CFI), Índices de Tucker-Lewis (TLI) e o Teste Qui-quadrado do modelo.

\section{Procedimentos Éticos}

Este estudo teve como base as regras descritas na Resolução 466/2012 do Conselho Nacional de Saúde (Brasil, 2012). A pesquisa foi aprovada sob o número CEP 13/1651, concedido pelo Comitê de Ética em Pesquisa da Universidade do Vale do Rio dos Sinos. Os participantes assinaram eletronicamente o TCLE ao marcar o item "sim". Os principais resultados da pesquisa foram enviados em forma de relatório para profissionais que registraram seu endereço de e-mail na página on-line da pesquisa.

\section{Resultados}

\section{Análise Fatorial Exploratória (AFE)}

Para a condução das análises fatoriais, foram identificadas as medidas de adequação dos dados para detecção da estrutura fatorial. Tanto o resultado do teste de $\mathrm{KMO}=0,84$, (Kaiser-Meyer-Olkin), quanto o do teste de esfericidade de Bartlett de $\left(\chi^{2}=698,83\right.$, $g l=62$ e $p<0,001)$, indicaram a viabilidade da fatorabilidade dos dados. O emprego da análise paralela, considerando 0,30 como valor de saturação para os itens, sugeriu três modelos e extração (com 10 fatores; com 11 e com 12 fatores).

Foi realizada uma AFE robusta por meio do método de extração Minimum Rank Factor Analysis (MRFA), com base na matriz de correlações policóricas dos itens da QETS, visando permitir a extração da proporção da variância comum explicada pelos fatores retidos. Com a finalidade de evitar a superestimação de fatores comuns, adotou-se a análise paralela como técnica estatística para interpretação dos fatores obtidos na AFE. Resultados similares foram obtidos entre aqueles produzidos pelo método de extração de componentes principais e a análise paralela, identificando, ao menos, 12 fatores principais.
Por meio do teste ANOVA, identificou-se que o modelo de 12 fatores possui maior poder explicativo, quando comparado aos demais (13 e14 fatores). Tal modelo sugeriu uma estrutura formada por 50 itens, divididos em 12 fatores pertencentes a quatro escalas. Após essa etapa, empregou-se a análise fatorial exploratória, utilizando o mesmo ponto de corte para os itens $(0,30)$. Informações detalhadas sobre a fatoração dos itens seguem descritas na Tabela 1 e na Tabela 2.

Os resultados da AFE apontam que os valores das cargas fatoriais variaram entre 0,312 e 0,999 e média de 0,620. A menor carga foi representada pelo item S13 da escala Personalidade e a maior identificada no item A14 da escala Antecedentes. O item A12 repetiu-se em dois fatores da escala Personalidade, apresentando carga positiva $(0,385)$ no fator Tipo de Tarefa e carga negativa $(-0,315)$ no fator Compreensão. Considerando a média dos valores por fator, a carga mais elevada foi obtida no fator Consequências Organizacionais $(0,797)$. Enquanto o índice mais baixo foi observado no fator Pressão Social $(0,406)$.

\section{Análise Fatorial Confirmatória}

A AFC revelou que o melhor modelo ajustado é composto por 12 fatores, recorrente também no estudo de Lara, Jiménez, Muñoz, Merino e Chávez (2011), embora com indicação de mudanças importantes na composição das escalas. Neste estudo, o ajuste ao modelo por meio da AFC utilizou análises estatísticas para a estimação dos dados e obteve os seguintes resultados: Root Mean Square Error of Approximation (RMSEA $=0,044 ; \mathrm{IC}=10 \%-90 \%$ ); Índice de Ajuste Comparativo $($ CFI $=0,949)$; Índices de Tucker-Lewis (TLI = 0,904); Teste Qui-quadrado do modelo $(7083,49)$ e Graus de Liberdade $(G L)=1176$; $p$-valor $<0,0001)$. Em termos comparativos, é possível identificar diferenças entre os índices de ajuste encontrados na análise fatorial confirmatória realizados no estudo de validação realizado por Lara et al. (2011), no contexto mexicano (RMSEA $=0,043 ; \mathrm{x} 2=83,3 ; \mathrm{CFI}=0,96 ;$ TLI $=0,90 ;$ GL $=$ $58 p<0,0001)$. Em ambos, entretanto, foi encontrado um modelo de 12 fatores. Neste estudo, a AFC sugere como melhor ajuste um conjunto de 50 itens distribuídos em doze fatores (Figura 1).

O modelo ajustado explicou $56 \%$ da variância acumulada (Tabela 3), cujo coeficiente de consistência interna foi de 0,91 (alfa de Cronbach). No estudo de Lara et al. (2011), o modelo ajustado representou $62 \%$ da variância acumulada e 0,78 de índice de consistência interna. 
Tabela 1.

Análise Fatorial Exploratória das Escalas Antecedentes e Trauma Secundário do QETS

\begin{tabular}{|c|c|c|c|c|c|c|c|c|c|c|}
\hline \multirow{2}{*}{ Escalas } & \multicolumn{10}{|c|}{ Componentes da Matriz Rotacional } \\
\hline & Itens & F1 & F2 & F3 & F4 & F5 & F6 & F7 & F8 & F9 \\
\hline \multirow{14}{*}{ Antecedentes } & A2 & 0,599 & & & & & & & & \\
\hline & A3 & 0,639 & & & & & & & & \\
\hline & A4 & 0,509 & & & & & & & & \\
\hline & A5 & 0,543 & & & & & & & & \\
\hline & A8 & & 0,412 & & & & & & & \\
\hline & A9 & & 0,365 & & & & & & & \\
\hline & C3 & & 0,442 & & & & & & & \\
\hline & A12 & & & 0,385 & & & & & & $-0,315$ \\
\hline & A14 & & & 0,999 & & & & & & \\
\hline & A15 & & & 0,447 & & & & & & \\
\hline & A16 & & & & 0,504 & & & & & \\
\hline & A17 & & & & 0,510 & & & & & \\
\hline & A18 & & & & 0,756 & & & & & \\
\hline & A19 & & & & 0,687 & & & & & \\
\hline \multirow{14}{*}{$\begin{array}{l}\text { Trauma } \\
\text { Secundário }\end{array}$} & A20 & & & & 0,730 & & & & & \\
\hline & S3 & & & & & 0,469 & & & & \\
\hline & S4 & & & & & 0,424 & & & & \\
\hline & S5 & & & & & 0,650 & & & & \\
\hline & $\mathrm{C} 2$ & & & & & 0,367 & & & & \\
\hline & $\mathrm{C} 4$ & & & & & 0,578 & & & & \\
\hline & C5 & & & & & 0,759 & & & & \\
\hline & C6 & & & & & 0,472 & & & & \\
\hline & $\mathrm{C} 7$ & & & & & 0,483 & & & & \\
\hline & S6 & & & & & & 0,501 & & & \\
\hline & S7 & & & & & & 0,533 & & & \\
\hline & S8 & & & & & & 0,696 & & & \\
\hline & S11 & & & & & & & 0,530 & & \\
\hline & S12 & & & & & & & 0,993 & & \\
\hline
\end{tabular}

Os fatores do modelo ajustado foram interpretados como: Desafio (F1), Estado de Humor (F2), Fadiga de Compaixão e Repercussões Sociais (F3), Satisfação em Ajudar (F4), Consequências Organizacionais (F5), Empatia (F6) Carga Horária e Laboral (F7), Trauma Secundário (F8), Compreensão (F9), Tipo de Tarefa (F10), Mudança de Crenças (F11) e
Pressão Social (F12). Os doze fatores estão distribuídos em quatro escalas que mantiveram a nomenclatura da versão original: Antecedentes (E1), Síndrome de Trauma Secundário (E2), Personalidade (E3) e Consequências (E 4).

Em relação ao estudo de validação de Lara et al. (2011), o estudo de análise da estrutura interna da 
Tabela 2.

Análise Fatorial Exploratória das Escalas Personalidade e Consequências do QETS

\begin{tabular}{|c|c|c|c|c|c|c|}
\hline \multirow{2}{*}{ Escalas } & \multicolumn{6}{|c|}{ Componentes da Matriz Rotacional } \\
\hline & Itens & F8 & F9 & F10 & F11 & F12 \\
\hline \multirow{18}{*}{ Personalidade } & S13 & 0,312 & & & & \\
\hline & P1 & 0,498 & & & & \\
\hline & $\mathrm{P} 2$ & 0,833 & & & & \\
\hline & P3 & 0,670 & & & & \\
\hline & P4 & 0,577 & & & & \\
\hline & P5 & & 0,642 & & & \\
\hline & P6 & & 0,401 & & & \\
\hline & P7 & & 0,616 & & & \\
\hline & P8 & & 0,356 & & & \\
\hline & P10 & & & 0,550 & & \\
\hline & P11 & & & 0,694 & & \\
\hline & P12 & & & 0,833 & & \\
\hline & P13 & & & 0,772 & & \\
\hline & P14 & & & 0,705 & & \\
\hline & P15 & & & & 0,819 & \\
\hline & P16 & & & & 0,692 & \\
\hline & P17 & & & & 0,71 & \\
\hline & P18 & & & & 0,862 & \\
\hline \multirow{3}{*}{ Conse-quências } & $\mathrm{C} 8$ & & & & & 0,788 \\
\hline & C9 & & & & & 0,804 \\
\hline & $\mathrm{C} 10$ & & & & & 0,779 \\
\hline
\end{tabular}

QETS revelou que: a escala Antecedentes conservou 14 itens da versão original e adicionou o item C3 no fator Pressão Social. Além deste, os outros três fatores compõem a escala (Carga Horária e Laboral, Tipo de Tarefa e Satisfação em Ajudar). Formada por três fatores, a escala Síndrome de Trauma Secundário manteve oito itens originais e acrescentou no fator Fadiga de Compaixão e Repercussões Sociais cinco itens (C2, C4, C5, C6 e C7), oriundos da escala Consequências. Os demais fatores mantiveram a nomenclatura (Mudança de Crenças e Sintomas de ETS).

Após o ajuste do item S13 na escala Personalidade, os fatores Desafio, Satisfação em Ajudar e Compreensão conservaram a estrutura original. Foram mantidos 17 itens e acrescido o item S13, da escala Síndrome de Trauma Secundário, no fator Empatia. Por fim, a escala Consequências passou a ser formada por um fator, composto pelos itens (C8, C9, C10), denominado Consequências Organizacionais. Cabe ressaltar que o ajuste do item S13 no fator Empatia, reduziu o número de itens do modelo para 49.

\section{Discussão}

O ETS compreende um significativo sofrimento que vem sendo identificado nos profissionais da saúde em diferentes países. No Brasil, há uma lacuna na produção científica acerca do tema, a qual toma maiores proporções se comparada ao crescente número de publicações sobre o ETS na literatura internacional (Dalagasperina, 2017). Dessa forma, o presente estudo examinou a estrutura fatorial do QETS, com vistas a utilizá-lo no país. O processo de ajuste ao modelo envolveu mudanças tanto na compreensão dos itens, quanto dos fatores. O item C3 (Tenho problemas cardíacos) se ajustou ao fator Pressão Social da escala Antecedentes. A ausência de congruência teórica para 


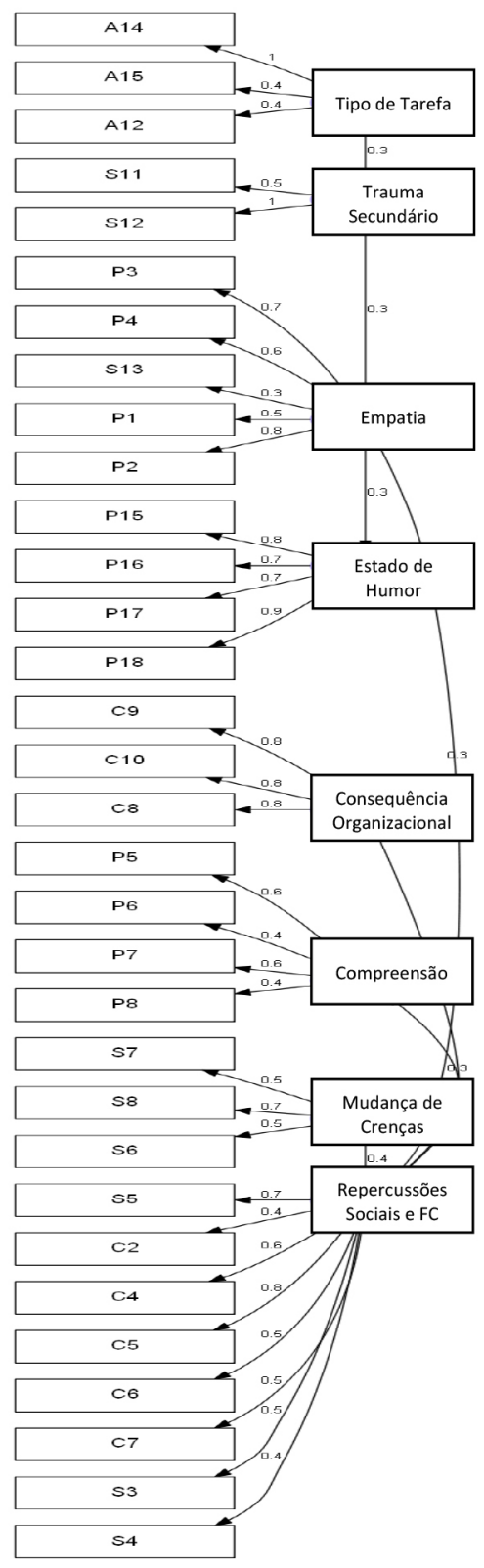

Figura 1. Análise fatorial exploratória.

item nessa escala, bem como sua baixa carga fatorial $(0,442)$ determinaram a remoção do item.

As implicações nos relacionamentos interpessoais (derivadas da escala Consequências) somadas aos itens da Fadiga de Compaixão do Fator Trauma Secundário, configuraram-se como manifestações da Síndrome de Trauma Secundário. Resultado similar foi observado na correlação entre os três tipos de Consequências (física, social e organizacional) e a Síndrome de Trauma Secundário, que indicaram associação positiva e com alto nível de significância entre as duas,

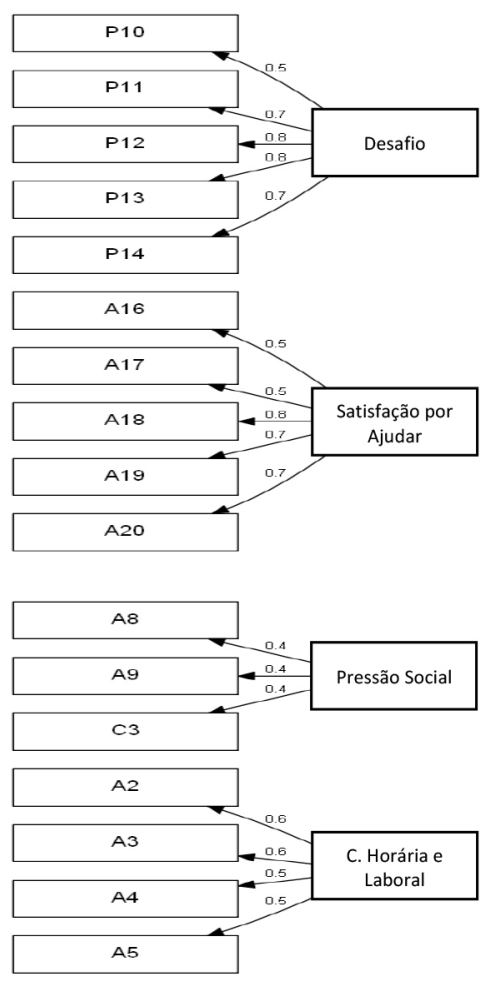

num estudo realizado com bombeiros e paramédicos (Meda et al., 2012).

Em relação as implicações negativas nos relacionamentos, níveis mais altos de ETS foram associados significativamente com: menor satisfação no relacionamento conjugal, redução da habilidade de comunicação social, aumento da evitação de pessoas e rupturas nos relacionamentos interpessoais (Bride, 2007; Dekel, Levinstein, Siegel, Fridkin, \& Svetlitzky, 2016; Lahav, Stein, \& Solomon, 2016; Robinson-Kilig, 2014). Essas alterações também podem estar associadas ao fato de 
Tabela 3.

Variancia das Escalas e Subescalas do QETS

\begin{tabular}{|c|c|c|c|c|c|}
\hline Escalas & Subescalas & Eigenvalues & $\begin{array}{c}\% \text { de } \\
\text { variância }\end{array}$ & $\begin{array}{c}\text { \% de variância } \\
\text { acumulada }\end{array}$ & $\begin{array}{c}\text { Variância } \\
\text { acumulada escala }\end{array}$ \\
\hline \multirow[t]{4}{*}{ Antecedentes } & Carga Horária e Laboral & 1,8 & 0,04 & 0,04 & \multirow[t]{4}{*}{0,15} \\
\hline & Pressão Social & 1 & 0,02 & 0,06 & \\
\hline & Tipo de Tarefa & 1,73 & 0,04 & 0,10 & \\
\hline & Satisfação em Ajudar & 2,59 & 0,05 & 0,15 & \\
\hline \multirow{3}{*}{$\begin{array}{l}\text { Síndrome } \\
\text { de Trauma } \\
\text { Secundário }\end{array}$} & $\begin{array}{l}\text { Fadiga de Compaixão e } \\
\text { Repercussões Sociais }\end{array}$ & 3,29 & 0,07 & 0,22 & \multirow[t]{3}{*}{0,15} \\
\hline & Mudança de Crenças & 1,97 & 0,04 & 0,26 & \\
\hline & Trauma Secundário & 1,85 & 0,04 & 0,3 & \\
\hline \multirow[t]{4}{*}{ Personalidade } & Empatia & 2,55 & 0,05 & 0,35 & \multirow[t]{4}{*}{0,21} \\
\hline & Compreensão & 1,83 & 0,04 & 0,39 & \\
\hline & Desafio & 2,78 & 0,06 & 0,45 & \\
\hline & Estado de Humor & 2,8 & 0,06 & 0,51 & \\
\hline Consequências & $\begin{array}{l}\text { Consequências } \\
\text { Organizacionais }\end{array}$ & 2,49 & 0,05 & 0,56 & 0,05 \\
\hline
\end{tabular}

Tabela 4

Coeficiente de Correlação de Pearson entre os fatores do QETS e o PCL-C

\begin{tabular}{lcccc}
\hline $\begin{array}{l}\text { Síndrome de Trauma Secundário } \\
\text { (para validar) }\end{array}$ & $\begin{array}{c}\text { PCL-C } \\
\text { Revivência }\end{array}$ & $\begin{array}{c}\text { PCL-C } \\
\text { Evitação }\end{array}$ & $\begin{array}{c}\text { PCL-C } \\
\text { Excitabilidade }\end{array}$ & $\begin{array}{c}\text { TOTAL } \\
\text { PCL-C }\end{array}$ \\
\hline Desafio & $-0,097$ & $-0,034$ & $-0,06$ & $-0,065$ \\
Estado de Humor & 0,198 & 0,263 & 0,272 & 0,267 \\
Fadiga de Compaixão e Repercussões Sociais & 0,68 & 0,726 & 0,732 & 0,773 \\
Satisfação em Ajudar & $-0,26$ & $-0,304$ & $-0,25$ & $-0,296$ \\
Consequências Organizacionais & 0,357 & 0,377 & 0,355 & 0,394 \\
Empatia & 0,436 & 0,433 & 0,467 & 0,481 \\
Carga horária e Laboral & 0,19 & 0,238 & 0,277 & 0,256 \\
Síndrome de Trauma Secundário & 0,373 & 0,349 & 0,338 & 0,381 \\
Compreensão & 0,507 & 0,531 & 0,45 & 0,538 \\
Tipo de Tarefa & 0,341 & 0,329 & 0,344 & 0,365 \\
Mudança de Crenças & 0,452 & 0,539 & 0,523 & 0,55 \\
Pressão Social & 0,36 & 0,431 & 0,328 & 0,408 \\
\hline
\end{tabular}

que os parceiros conjugais de pessoas acometidas pelo ETS tendem a apresentar maior nível de sofrimento psíquico, caracterizado por sintomas de estresse pós-traumático ansiedade e depressão (Dekel et al., 2016).

O entendimento da Fadiga de Compaixão como manifestação do ETS encontra respaldo na literatura.
Figley (1995) utilizou o termo para descrever os efeitos do estresse traumático em profissionais de saúde que trabalham com pacientes traumatizados. A Fadiga de Compaixão se refere à exaustão física e emocional e é compreendida como um termo genérico por envolver uma combinação de sintomas do ETS e da SB. (Newell 
\& MacNeil, 2010). Manifesta-se comumente entre os em profissionais de saúde, particularmente naqueles que cuidam de pacientes crônicos e/ou agudamente doentes (Klein, 2018).

$\mathrm{Na}$ escala Personalidade, o item A12 (Fico muito aborrecido quando atendo alguém que sofreu maus tratos) concorreu em dois fatores (Tipo de Tarefa e Compreensão). Os demais itens do Tipo de Tarefa estão relacionados ao atendimento às vítimas de violência e ao sofrimento envolvido nesse processo, enquanto o fator Compreensão corresponde a falta de clareza cognitiva na tomada de decisões e nas tarefas a serem desempenhadas (Moreno et al., 2004a).

A pontuação negativa do item A12 no fator Compreensão denota que o profissional não se aborrece ao atender pessoas que sofreram maus tratos. Desse modo, isso estaria associado a uma distorção cognitiva frente ao sofrimento dos pacientes. Tal vivência é característica da Fadiga de Compaixão que é definida como uma redução da capacidade ou do interesse do profissional em ser empático ou suportar o sofrimento dos pacientes (Figley, 1995). Frente a esse entendimento, optou-se por manter o item em seu fator original (Tipo de Tarefa), em função de apresentar maior congruência teórica e carga fatorial mais elevada.

A menor carga fatorial foi observada no item S13 (Guardo imagens muito reais daqueles acidentes que mais me afetaram) que migou para o fator Empatia. A empatia é o principal recurso utilizado pelos profissionais para compreender o processo traumático vivenciado pelo paciente (Figley, 1995; Moreno-Jiménez et al., 2004b). Quanto maior for o nível de envolvimento afetivo, maior será a probabilidade de desencadear os sintomas do transtorno, como nesse caso, a revivência (Figley, 1995). Correlações positivas e significativas foram encontradas entre a empatia e as três subescalas da Síndrome de Trauma Secundário em um estudo que correlacionou as quatro escalas do Modelo Processual do ETS (Meda, Moreno-Jiménez, Palomera, Arias, \& Vargas, 2012).

O item com maior carga fatorial $(0,999)$ foi o A14 (Custa-me esquecer situações que a vítima é menor de idade ou idoso). Esse resultado corrobora com estudos internacionais que apontam como fatores desencadeantes de estresse entre os profissionais que atuam em emergências e cuidados paliativos, as situações de abuso e a idade do paciente (Berg, Harshbarger, Ahlers-Schmidt, \& Lippoldt, 2016; West, 2017). Ao final da análise o ajuste ao modelo passa a ser composto por 48 itens, uma vez que o item C3 foi removido, e o item A12 (que apresentou sobreposição) foi ajustado ao fator Tipo de Tarefa.

Um estudo de validação da escala Síndrome de Trauma Secundário do QETS, realizado no México com profissionais de emergências e bombeiros, obteve dados satisfatórios. A escala manteve os itens e sofreu apenas uma alteração. O item 3, do fator Trauma Secundário, apresentou maior congruência teórica no fator Fadiga de Compaixão (Meda et al., 2011). Tal resultado distancia-se dos achados deste estudo, no qual foram eliminados seis itens da escala original e acrescidos cinco itens da escala Consequências. Essa distinção pode ser atribuída ao fato de que as análises dessa validação envolveram todas as escalas do QETS e foram realizadas com profissionais de outras categorias.

Foram identificadas correlações significativas entre as versões do QETS e do PCL-C que indicam a convergência entre as escalas. Tal resultado sugere que ambos os instrumentos mensuram o mesmo traço latente (Pasquali, 2003). A pontuação negativa no fator Desafio se deve ao fato de que os itens estão associados à aspectos positivos, assim como ocorre na Satisfação em Ajudar. A ausência de significância nesse fator pressupõe que encarar o trabalho como um desafio não se configura como um fator de proteção ou risco para o desenvolvimento do ETS, neste estudo.

\section{Considerações Finais}

Este estudo examinou a estrutura interna do Questionário de Estresse Traumático Secundário para uso no país com profissionais da saúde. Os resultados das análises demostraram índices psicométricos relativamente baixos e uma nova organização dos itens das escalas do QETS, apontando um distanciamento teórico da versão original do instrumento, diferentemente do estudo de Lara et al. (2011), que indicou propriedades psicométricas satisfatórias da CETS para a população mexicana. Isso pressupõem que ambos não mensuram da mesma forma o constructo ETS, baseado no Modelo Processual.

Como limitações deste estudo, cabe ressaltar que os resultados obtidos, embora objetivando a representatividade nacional, foram, em sua maioria, fornecidos por profissionais da região Sul, sendo a quase totalidade deles do sexo feminino. Outro limitador refere-se ao fato de que a investigação envolveu diferentes categorias profissionais da área da saúde, sendo que a maioria era da enfermagem. A ausência de estudos de validação realizados com as quatro escalas do CETS limitou a 
discussão dos dados. Sugere-se que novos estudos possam utilizar esse instrumento e ampliar sua validação para outros tipos de amostras. Além disso, a diferença entre a fatoração dos dados pode estar associada à ausência de estudos quase-experimentais que pudessem identificar o estresse traumático secundário em uma amostra clínica.

Almeja-se que o conhecimento acerca dessa forma de adoecer possa ser disseminado, tanto no meio acadêmico quanto na clínica, bem como nas diferentes instituições de saúde do país. A identificação do processo do ETS em diferentes contextos pode elucidar formas de prevenção e de tratamento desse transtorno. Sugere-se que novos estudos psicométricos analisem as escalas do questionário, separadamente, e envolvam outras categorias profissionais, especialmente aquelas dos serviços de emergências.

\section{Referências}

Associação Americana de Psiquiatria. (2013). Manual diagnóstico e estatístico de transtornos mentais: DSM-V. (5 $5^{\mathrm{a}}$ ed.) Porto Alegre: Artmed.

Barbosa, S. da. C., Souza, S., \& Moreira, J. S. (2014. A fadiga por compaixão como ameaça à qualidade de vida profissional em prestadores de serviços hospitalares. Revista Psicologia: Organizações e TrabaIho, 14(3), 315-332. Recuperado de http://pepsic. bvsalud.org/scielo.php?script=sci_arttext\&pid =S1984-66572014000300007

Beaton, R. D., \& Murphy, S. A. (1995). Working with people in crisis: Research implications. Em C. R. Figley (Ed.), Compassion fatigue: Coping with secondary traumatic stress disorder in those who treat the traumatized, (pp. 51-81). Nueva York: Brunner/ Mazel.

Berg, M., Harshbarger, J. L., Ahlers-Schmidt, C. R., Lippoldt, D. (2016). Exposing compassion fatigue and burnout syndrome in a trauma team: A qualitative study. Journal of Trauma Nursing, 23(1), 3-10. doi: 10.1097/JTN.0000000000000172

Berger, W., Mendlowicz, M. V., Souza, W. F., \& Figueira, I. (2004). Equivalência semântica da versão em português da Post-Traumatic Stress Disorder Checklist - Civilian Version (PCL-C) para rastreamento do transtorno de estresse pós-traumático. Revista de Psiquiatria do Rio Grande do Sul, 26, 167175. doi:10.1590/S0101- 81082004000200006
Brasil. Conselho Nacional de Saúde. Resolução no 466 de 12 dezembro de 2012. Diário Oficial da República Federativa do Brasil. Brasil (DF). 12 de dezembro de 2012.

Bride, B. E., Radey, M., \& Figley, C. R. (2007). Measuring compassion fatigue. Clinical Social Work. Journal, 35, 155-163. doi: http://dx.doi.org/10 .1007/ s10615-007-0091-7

Carlotto, M. S., \& Câmara, S. G. (2008). Análise de produção científica de burnout no brasil. Revista Psico, 39(2), 152-158. Recuperado de http:// revistaseletronicas.pucrs.br/fass/ojs/index.php/ revistapsico/article/view/1461

Castro, E. K. D., Massom, T., \& Dalagasperina, P. (2018). Estresse traumático secundário em psicólogos. Revista Psicologia e Saúde, 10(1), 115-125. doi: 10.20435/pssa.v9i3.554

Cieslak. R., Shoji, K., Allison, D., Melville, E., Luszczynska, A., \& Benight, C. C. (2014). A meta-analysis of the relationship between job burnout and secondary traumatic stress among workers with indirect exposure to trauma. Psychological Services, 11(1), 75 86. doi: $10.1037 / \mathrm{a} 0033798$

Dekel, R., Levinstein, Y., Siegel, A., Fridkin, S., \& Svetlitzky, V. (2016). Secondary traumatization of partners of war veterans: The role of boundary ambiguity. Journal of Family Psychology, 30(1), 63-71. doi: 10.1037/fam0000163

Dutton, M. A., \& Rubenstein, F. L. (1995). Working with people with PTSD: Research implications. Em C. R. Figley (Ed.), Compassion fatigue: Coping with secondary traumatic stress disorder in those who treat the traumatized (pp. 82-100). Nueva York: Brunner/ Mazel

Figley, C. R. (1995). Compassion Fatigue: Coping with secondary traumatic stress disorder in those who treat the traumatized. Nueva Yoork: Brunner/Mazel Publishers.

Figley, C. R.(dir.). (1983). The family as victim: Mental health implications. Em P. Berner (dir.) Procedings of the VII World Congress of Psychiatry. London: Plenum.

Galiana, L., Arena, F., Oliver. A., Sansó, N., \& Benito, E. (2017). Compassion satisfaction, compassion fatigue, and burnout in Spain and Brazil: ProQOL validation and cross-cultural diagnosis. Journal of 
Pain and Symptom Management 53(3), 598-604. doi: 10.1016/j.jpainsymman.2016.09.014

Hair, J. F., Anderson, R. E., Tatham, R. L., \& Black, W. C.(2007) Análise multivariada de dados. Porto Alegre: Bookman.

Hayton, J. C., Allen, D. G., \& Scarpello, V. (2004). Factor retention decisions in exploratory factor analysis: A tutorial on parallel analysis. Organizational research methods, 7(2), 191-205. doi: $10.1177 / 1094428104263675$

Hensel, J. M., Ruiz, C., Finney, C. \& Dewa, C. D. (2015). Meta-analysis of risk factors for secondary traumatic stress in therapeutic work with trauma victims. Journal of Traumatic Stress, 28(2), 83-91. doi:10.1002/jts.21998

International Society for Traumatic Stress Studies. (2014). Assessing trauma - Posttraumatic Stress Disorder Checklist (PCL). Deerfield, IL: Author. Recuperado de http://www.istss.org/ZAM/Template. cfm?Section $=$ Home

Kintzle, S., Yarvis, J. S., \& Bride, B. E. (2013). Secondary traumatic stress in military primary and mental health care providers. Military Medicine, 178, 13101315. doi: 10.7205/MILMED-D-13-00087

Klein C. J., Riggenbach-Hays, J. J., Sollenberger, L. M., Harney, D. M. \& McGarvey JS1. (2018). Quality of Life and Compassion Satisfaction in Clinicians: A Pilot Intervention Study for Reducing Compassion Fatigue. American Journal of Hospice \& Palliative Medicine, 35(6),882-888

Lahav, Y., Stein, J. Y., \& Solomon, Z. (2016). Keeping a healthy distance: Self-differentiation and perceived health among ex-prisoners-of-war's wives. Journal of Psychosomatic Research, 89(1), 61-68. doi: 10.1016/j.jpsychores.2016.08.008

Lara, R. M. M., Jiménez, B. M., Muñoz, A. R., Merino, E. D. A., \& Chávez, A. P. (2011). Validación mexicana de la escala de estrés traumático secundario. Psicología y salud, 21(1), 5-15. doi:10.25009/ pys.v21i1.581

Lima, E. P., \& Ávila, A. A. (2011). Prevalência e fatores associados ao Transtorno de Estresse Pós-Traumático (TEPT) em profissionais de emergência: Uma revisão sistemática da literatura. Revista Brasileira de Epidemiologia, 14(2), 217-30. doi: 10.1590/ S1415-790X2011000200004
Meda, R., Moreno-Jiménez, B., Muñoz, A. R., Merino, E. D. A. \& Chávez, A. P. (2011). Validación mexicana de la Escala de Estrés Traumático Secundario Mexican validation of the Secondary Traumatic Stress Scale. Psicología y Salud, 21(1), 5-15.

Meda, R., Moreno-Jiménez, B., Palomera, S., Arias, E., \&Vargas, R. (2012). La Evaluación del Estrés Traumático Secundario. Estudio Comparado en Bomberos y Paramédicos de los Servicios de Emergencia de Guadalajara, México. Terapia Psicológica, 30(2), 31-41. doi: 10.4067/ S0718-48082012000200003

Moreno-Jiménez, B., Morante, M. E., Rodríguez, M., A., \& Garrosa, E. (2004a). Secondary Traumatic Stress as Psychosocial Emergent Risk. Paper presented at the 18th Annual Conference of the European Health Psychology Society, Helsinki.

Moreno-Jiménez, B., Morante, M. E., Garrosa, E. \& Rodriguez-Carvajal R. (2004b). Estrés Traumático Secundario: el coste del cuidar el trauma. Psicología Condutual, 12(2), 215-231. Recuperado de http:// www.bago.com/BagoArg/Biblio/psiqweb269. htm

Newell, J. M., \& MacNeil, G. A. (2010). Professional burnout, vicarious trauma, secondary traumatic stress, and compassion fatigue: A review of theoretical terms, Risk Factors, and Preventive Methods for Clinicians and Researchers. Best Practice in Mental Health, 6(2), 57-68. Recuperado de http:// ezp.waldenulibrary.org/login?url=http:// search. ebscohost.com/login. aspx?direct $=$ true $\& \mathrm{db}=\mathrm{a} 9 \mathrm{~h}$ $\& \mathrm{AN}=60132515 \&$ scope $=$ site

Pasquali, L. (2003). Psicometria: Teoria dos testes na psicologia e na educação. Petrópolis: Vozes.

Pedras, C. S., \& Pereira, M. G. (2012). Exposição directa e indirecta ao trauma: Perturbação de stress pós-traumático (PTSD) e perturbação de stress traumático secundário (STSD). Psicologia na Actualidade, 6, 19-37. Recuperado de https://repositorium. sdum.uminho.pt/bitstream/1822/22272/1/revista $\% 20$ psi\%20naatualidade.pdf

Perkins, E. B., \& Sprang, G. (2013). Results from the Pro-QOL-IV for substance abuse counselors working with offenders. International Journal of Mental Health and Addiction, 11, 199-213. doi: 10 .1007/ s11469-012-9412-3 
Robinson-Keilig, R. A. (2014). Secondary traumatic stress and disruptions to interpersonal functioning among mental health therapists. J Interpers Violence, 29(8), 1477-96. doi: 10.1177/0886260513507135

Tabachnick, B., \& Fidell, L. (2007). Using multivariate analysis. Needham Heights: Allyn \& Bacon.

West, B. O. (2016). Quality of Life among South African Informal, Non-familial Caregivers of Childhood
Sexual Abuse Survivors (Doctoral dissertation, The Chicago School of Professional Psychology). Recuperado de https://search.proquest.com/ docview/1794167682?pq-origsite $=$ gscholar

Recebido em: 08/10/2018 Reformulado em: 31/03/2020

Aprovado em: 09/07/2020 
Sobre os autores:

Patricia Dalagasperina é Graduada em Psicologia pela Universidade Regional Integrada do Alto Uruguai e das Missões, possui Especialização em Terapia Cognitivo Comportamental pelo Instituto Cognitivo, realizou Mestrado e Doutorado em Psicologia Clínica na Universidade do Vale do Rio dos Sinos, fez estágio pós doutoral em Avaliação e Desenvolvimento na Universidade Federal de Santa Catarina.

ORCID: https://orcid.org/0000-0001-8752-3856

E-mail:pati-d@hotmail.com

Elisa Kern de Castro possui graduação em Psicologia, mestrado em Psicologia do Desenvolvimento doutorado em Psicologia Clínica e da Saúde - Universidad Autónoma de Madrid e estágio pós-doutoral em Psicologia pela Universidad de Salamanca. Foi coordenadora executiva do PPG Psicologia Unisinos. Atualmente é professora na Universidade Lusíada de Lisboa no Mestrado em Psicologia Clínica. Faz parte do CLISSIS.

ORCID: https://orcid.org/0000-0002-1290-7561

E-mail: elisa.kerndecastro@gmail.com

Roberto de Moraes Cruz é Psicólogo, especialista em avaliação psicológica, ergonomia e psicologia ocupacional, doutor em Engenharia de Produção, pós-doutorado em Métodos e Diagnóstico, Medicina Nuclear e Episemiologia. Atualmente é professor e pesquisador do Departamento e do Programa de Pós-Graduação em Psicologia da Universidade Federal de Santa Catarina. Líder do Laboratório Fator Humano (UFSC) e pesquisador do Núcleo de Pesquisa em Neuropsicologia e Saúde.

ORCID: https://orcid.org/0000-0003-4671-3498

E-mail: robertocruzdr@gmail.com

Artur Pereira é Graduado em Economia e Ciência Política pela Universidade de Toronto - Campus St.George. Master of Business Administration pela Fundação Getúlio Vargas.

ORCID https://orcid.org/0000-0003-4016-3341

E-mail: arturcp@gmail.com

Bernardo Moreno Jiménez é Graduado em Psicologia pela Universidade de Leuven. É Doutor em Psicologia pela Universidade Autonama de Madrid. Atualmente é professor credenciado em Psicologia da Personalidade, com ênfase em processos de personalidade, estresse e saúde, e em Psicologia da Saúde Ocupacional, com ênfase em riscos psicossociais e psicopatologia clínica ocupacional. Diretor do Título Próprio Intervenção Psicossocial em Emergências, crise e catástrofe.

ORCID: https://orcid.org/0000-0001-5360-0518

E-mail: bernardo.moreno@uam.es

Contato com os autores:

Universidade Federal de Santa Catarina, Departamento de Ciências Humanas, Laboratório Fator Humano Rua Engenheiro Agronômico Andrei Cristian Ferreira, s/n, Trindade

Florianópolis-SC, Brasil

CEP: 88040-900 